\title{
ON NEF REDUCTIONS OF PROJECTIVE IRREDUCIBLE SYMPLECTIC MANIFOLDS
}

\author{
DAISUKE MATSUSHITA
}

\begin{abstract}
Let $X$ be a projective irreducible symplectic manifold and $L$ is a non trivial nef divisor on $X$. Assume that the nef dimension of $L$ is strictly less than the dimension of $X$. We prove that $L$ is semiample.
\end{abstract}

\section{INTRODUCTION}

We begin with the definitions of irreducible symplectic manifolds and Lagrangian fibrations.

Definition 1.1. A compact Kähler manifold $X$ is said to be a symplectic manifold if $X$ carries a holomorphic symplectic form. Moreover if $X$ satisfies the following two conditions, $X$ is said to be irreducible.

(1) $\pi_{1}(X)=\{1\}$

(2) $h^{2}\left(X, \mathcal{O}_{X}\right)=1$.

Definition 1.2. Let $X$ be a symplectic manifold, $\omega$ a symplectic form on $X$ and $S$ a normal variety. A proper surjective morphism with connected fibres $f:(X, \omega) \rightarrow$ $S$ is said to be a Lagrangian fibration if a general fibre $F$ of $f$ is a Lagrangian variety with respect to $\omega$, that is, $\operatorname{dim} F=(1 / 2) \operatorname{dim} X$ and the restriction of the symplectic 2-form $\left.\omega\right|_{F}$ is identically zero.

The simplest example of an irreducible symplectic manifold is a $K 3$ surface. It is expected that $K 3$ surfaces and irreducible symplectic manifolds share many geometric properties. Let $L$ be a non trivial nef divisor on a $K 3$ surface. It is well known that if $L^{2}=0$, then $L$ defines an elliptic fibration, which is the simplest example of a Lagrangian fibration. By using a Beauville-Bogomolov-Fujiki form $q$ [3, Théorème 5], we can consider the following problem for an irreducible symplectic manifold, which is posed by Huybrechts and Sawon:

Problem 1.3. Let $X$ be an irreducible symplectic manifold and $L$ a non trivial nef divisor on $X$. If $q(L)=0$, then $L$ defines a Lagrangian fibration?

1991 Mathematics Subject Classification. Primary 14E30, Secondary 14J40.

* Partially supported by Grand-in-Aid \# 15740002 (Japan Society for Promortion of Sciences). 
We give a partial answer for the above problem. To state our results, we introduce the nef dimension of a nef divisor, which is due to [2].

Definition-Theorem 1.4. [2, Theorem 2.1 and Definition 2.7]

Let $X$ be a normal projective variety and $L$ a nef divisor on $X$. Then there exists a rational map $f: X \rightarrow S$ which satisfies the following three conditions:

(1) A general fibre of $f$ is compact.

(2) $L$ is numerically trivial on a general fibre of $f$.

(3) For every general point $x \in X$ and every irreducible curve passing through $x$ with $\operatorname{dim} f(C)>0$, we have L.C $>0$.

Moreover the map $f$ is unique up to birational equivalence of $S$. We define the nef dimension of $L$ as

$$
n(L):=\operatorname{dim} S
$$

Our result is the following:

THEOREM 1.5. Let $X$ be a projective irreducible symplectic manifold and $L$ a non trivial nef divisor on $X$. Assume that $n(L)<\operatorname{dim} X$. Then $L$ is semiample, that is, there exists an integer $M$ such that the linear system $|M L|$ is free.

REMARK 1.6. Since the morphism $f: X \rightarrow S$ which is defined by $|M L|$ satisfies the three conditions of Definition-Theorem 1.4, we have $\operatorname{dim} S=n(L) . \quad B y[9$, Theorem 2] and [9, Theorem 1], $f$ is a Lagrangian fibration.

REMARK 1.7. Let $L$ be a nef divisor on a projective variety. By [2, Proposition 2.8], we have

$$
n(L) \geq \nu(L) \geq \kappa(L),
$$

where $\kappa(L)$ is the Kodaira dimension of $L$ and $\nu(L)$ is the numerical Kodaira dimension of $L$. Thus the assumption $n(L)<\operatorname{dim} X$ implies $\nu(L)<\operatorname{dim} X$. We note that if $X$ is an rreducible symplectic manifold and $L$ a nef divisor on $X$, then $q(L)=0$ if and only if $\nu(L)<\operatorname{dim} X$ by [5, Theorem 4.7].

REMARK 1.8. If we consider an irreducible symplectic manifold which is the moduli space of semi stable torsion free sheaves on a K3 surface or an abelian surface, then we have other existence conditions of fibre space structures. Please see [6, Theorem 1.3], [8, Theorem 4.3] and [11, Theorem 2]. On the other hand, Amerik and Campana study a rational map $f: X \rightarrow S$ from an irreducible symplectic manifold $X$ to a normal variety $S$ such that $0<\operatorname{dim} S<\operatorname{dim} X$ and the Kodaira dimension of a general fibre of $f$ is zero. Please see [1, Théorème 3.6].

Acknowledgements. The author express his thanks to Professor O. Fujino who informed me the nef dimension. He also express his thanks to Professor 
E. Amerik who sended me a copy of her preprint. By that article, the proof of Theorem 1.5 is simplified. He also express his thanks to Professor S. Boucksom who gave me an important comment of Claim 2.4.

\section{Proof of Theorem 1.5}

2.1. By [7, Theorem 1], it is enough to prove that

$$
\nu(L)=\kappa(L) .
$$

By the assumption, there exists a rational map $f: X \rightarrow S$ which satisfies the three conditions of Definition-Theorem 1.4. Let $\pi: Y \rightarrow X$ be a resolution of indeterminacy of $f$. We denote by $g$ the induced morphism.

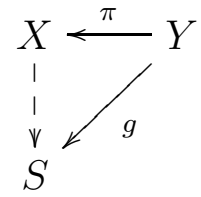

Claim 2.2. Let $L_{S}$ be a very ample divisor on $S$. Then

$$
q\left(\pi_{*} g^{*} L_{S}\right)=0 .
$$

Proof. Let $H_{1}$ and $H_{2}$ be general members of the linear system of $g^{*} L_{S}$. By $[3$, Theorem 5],

$$
q\left(\pi_{*} g^{*} L_{S}\right)=\frac{\operatorname{dim} X}{2} \int_{X} \pi_{*} H_{1} \pi_{*} H_{2}(\omega \bar{\omega})^{(1 / 2) \operatorname{dim} X-1},
$$

where $\omega$ is a symplectic form on $X$. Since $L_{S}$ is very ample, the intersection $\pi_{*} H_{1}$ and $\pi_{*} H_{2}$ defines a codimension 2 effective cycle. Thus $q\left(\pi_{*} g^{*} L_{S}\right) \geq 0$. We derive a contradiction assuming that $q\left(\pi_{*} g^{*} L_{S}\right)>0$. Let $\mathcal{P}$ be the positive cone attached to $q$ and $\mathcal{E}$ the pseudo effective cone. By [4, Theorem $4.3(\mathrm{i})], \mathcal{P} \subset \mathcal{E}$. Hence $\pi_{*} g^{*} L_{S}$ defines an interior point of $\mathcal{E}$. This implies $\pi_{*} g^{*} L_{S}$ is big. Let $F$ be a general fibre of $g$. Then $\left.\pi^{*} \pi_{*} g^{*} L_{S}\right|_{F}$ is big. Since $\pi$ is isomorphic in some neighbourhood of $F$, $\left.\pi^{*} \pi_{*} g^{*} L_{S}\right|_{F}=\left.g^{*} L_{S}\right|_{F}=0$. That is a contradiction.

Claim 2.3.

$$
q\left(L, \pi_{*} g^{*} L_{S}\right)=0
$$

Proof. Since $L$ is nef, $q\left(L, \pi_{*} g^{*} L_{S}\right) \geq 0$. We compute $q\left(L+\pi_{*} g^{*} L_{S}\right)$. By the assumption and Remark1.7, $q(L)=0$. By Claim 2.2, $q\left(\pi_{*} g^{*} L_{S}\right)=0$. Thus

$$
q\left(L+\pi_{*} g^{*} L_{S}\right)=2 q\left(L, \pi_{*} g^{*} L_{S}\right) .
$$

If we assume $q\left(L, \pi_{*} g^{*} L_{S}\right)>0$, then we have $L+\pi^{*} g_{*} L_{S}$ is big by the same argument in the proof of Claim 2.2. However

$$
\left.\pi^{*}\left(L+\pi_{*} g^{*} L_{S}\right)\right|_{F}=\pi^{*} L \equiv 0
$$


by the condition (2) of Definition-Theorem 1.4. That derives a contradiction.

Claim 2.4. There exists a positive constant $\lambda_{0}$ such that

$$
\lambda_{0} L \sim_{\mathbb{Q}} \pi_{*} g^{*} L_{S}
$$

Proof. Let $L^{\vee}$ be the hyperplain in $H^{1,1}(X, \mathbb{C})_{\mathbb{R}}$ defined by $q(L, *)$. Assume that $L^{\vee}$ contains an interior of the positive cone $\mathcal{P}$ of $X$. Then there exists a divisor $H$ such that $q(H)>0$ and $q(L, H)<0$. However this derives a contradiction because the signature of $q$ on $H^{1,1}(X, \mathbb{C})_{\mathbb{R}}$ is $(1, k-1)$, where $k=\operatorname{dim} H^{1,1}(X, \mathbb{C})_{\mathbb{R}}$. Hence $L^{\vee}$ intersects only the boundary of the closure $\overline{\mathcal{P}}$ of $\mathcal{P}$. Since $\mathcal{P}$ is a quadratic cone, $L^{\vee} \cap \overline{\mathcal{P}}$ coincides with the ray which is generated by $L$. By Claim $2.3, L$ and $g^{*} L_{S}$ are contained in the same ray.

2.5. Proof of Theorem 1.5. By Claim 2.4, we obtain

$$
\lambda_{0} \pi^{*} L \sim_{\mathbb{Q}} g^{*} L_{S}+\sum e_{i} E_{i}
$$

where $E_{i}$ is a $\pi$-exceptional divisor. Hence we have the following inequalities:

$$
\nu(L) \geq \kappa(L) \geq \kappa\left(g^{*} L_{S}\right)=n(L) .
$$

By the inequality in Remark 1.7, we are done.

\section{REFERENCES}

[1] E. Amerik and F. Campana, Fibations méromorphe sur certaines variétés à fibré trivial, mathAG/0510299.

[2] T. Bauer, F.Campana, T. Eckl, S. Kebekus, T. Peternell, S. Rams, T. Szemberg and L. Wotzlaw, A reduction map for nef line bundles, Complex geometry (Göttingen, 2000) Springer, Berlin, 27-36.

[3] A. Beauville, Variétés Kähleriennes dont la première classe de Chern est nulle, J. Differential. Geom., 18, (1983), 755-782.

[4] S. Boucksom, Divisorial Zariski decompositions on compact Kähler manifolds, Ann. Scient. Éc. Norm. Sup., 37, (2004), 45-76.

[5] A. Fujiki, On the de Rham cohomology group of a compact Kähler symplectic manifold, Algebraic geometry, Sendai, 1985, Adv. Stud. Pure Math., 10, 105-165.

[6] M, Gulbrandsen, Lagrangian fibrations on generalized Kummer varieties, mathAG/0510145.

[7] Y. Kawamata, Pluricanonical sysmtems on minimal algebraic varieties, Invent. Math., 79, (1985), no. 3, 567-588.

[8] D. Markshevich, Rational lagrangian fibrations on punctual hilbert scheme of points of K3 surfaces, mathAG/0509346.

[9] D. Matsushita, On fibre space structures of a projective irreducibe symplectic manifold, Topology, 38 (1999), 79-83.

[10] _ Addendum to: On fibre space structures of a projective irreducible symplectic manifold, Topology, 40 (2001), 431-432.

[11] J. Sawon, Lagrangian fibrations on Hilbert schemes of points on K3 surfaces , mathAG/0509224. 
Division of Mathematics, Graduate School of Science, Hokkaido University, SAPPORO, 060-0810 JAPAN

E-mail address: matusita@math.sci.hokudai.ac.jp 